\title{
Article \\ Surface-Enhanced Raman Spectroscopy for the Investigation of Chromogenic Motion Picture Films: A Preliminary Study
}

\author{
Margherita Longoni *(D), Francesco Ferretti (D), Sofia Zucca, Letizia Caielli and Silvia Bruni *(D) \\ Department of Chemistry, Università degli Studi di Milano, via C. Golgi 19, 20133 Milano, Italy; \\ francesco.ferretti@unimi.it (F.F.); sofia.zucca@studenti.unimi.it (S.Z.); letizia.caielli@studenti.unimi.it (L.C.) \\ * Correspondence: margherita.longoni@unimi.it (M.L.); silvia.bruni@unimi.it (S.B.)
}

check for

updates

Citation: Longoni, M.; Ferretti, F.; Zucca, S.; Caielli, L.; Bruni, S. Surface-Enhanced Raman Spectroscopy for the Investigation of Chromogenic Motion Picture Films: A Preliminary Study. Chemosensors 2022, 10, 101. https://doi.org/ $10.3390 /$ chemosensors 10030101

Academic Editor: Lucio Litti

Received: 31 January 2022

Accepted: 3 March 2022

Published: 6 March 2022

Publisher's Note: MDPI stays neutral with regard to jurisdictional claims in published maps and institutional affiliations.

Copyright: (C) 2022 by the authors. Licensee MDPI, Basel, Switzerland. This article is an open access article distributed under the terms and conditions of the Creative Commons Attribution (CC BY) license (https:// creativecommons.org/licenses/by/ $4.0 /)$.

\begin{abstract}
In the present work, surface-enhanced Raman spectroscopy (SERS) is proposed for the identification of dyes in chromogenic films. These substances, which are generated within the film itself during the colour development process, are first studied on reference molecules synthesised for the purpose and, subsequently, on dyes extracted from a chromogenic film. SERS spectroscopy proved to be an efficient technique for their investigation, providing a proof of concept for its applicability for further studies on this complex topic. SERS spectra of the reference molecules were also compared with normal Raman spectra, acquired with a spectrometer based on SSE ${ }^{\mathrm{TM}}$ technology. Furthermore, the excellent SERS properties of anisotropic nanomaterials, such as silver nanostars, have been confirmed.
\end{abstract}

Keywords: SERS; chromogenic motion picture films; dyes; silver nanostars; SSE ${ }^{\mathrm{TM}}$ Raman

\section{Introduction}

In the last century, photographic and motion picture film materials have enriched our cultural heritage. At the beginning, colours were applied on every single frame of the film by hand or with special stencils. Subtractive synthesis was then developed: the chromatic tone was initially obtained with the dye-transfer process, i.e., adding from one to three layers of colour on the film. In this regard, it is worth mentioning the Technicolor process $\mathrm{n}$. IV which provides that three black-and-white negative films coloured by imbibition with magenta, cyan and yellow dyes, are printed on a film coated with a substance capable of absorbing the dyes. Finally, chromogenic Monopacks were introduced, where the colourforming substances are divided into several layers of the emulsion, and are still in use nowadays [1]. These motion picture films, despite being quite recent, tend to degrade due to environmental conditions (i.e., humidity, temperature, exposure to light) and the main problem concerns the loss of colour due to the degradation of the dye. Currently digital restoration is the only means to restore the original appearance of films, but it requires the formulation of hypotheses on the evolution of the degradation processes as a function of time, with the aim of obtaining information on the initial colorimetric coordinates. In this scenario, a very limited number of studies have been reported on the identification of colouring substances in film materials [2,3]. In this regard, it is worth noting that motion picture films have a very complex structure, and the chemical mechanism underlying colour formation is also complex. For simplicity, the general stratigraphy of a film can be summarised as follows: a layer of polymeric support (the thickest one), typically cellulose triacetate or polyester; an image layer consisting of a photosensitive gelatin emulsion, which contains the substances responsible for the colours; an anti-halo undercoat and a final coat for protective purposes. In the case of black-and-white images, the hues are given by silver halide crystals which turn into silver during processing. Referring instead to the colour images, the emulsion is divided into three layers (each 5-10 $\mu \mathrm{m}$ thick), alternating with as many filtering layers and each containing magenta, yellow, or cyan dye. Together, 
they determine the final colouring. In such systems, the three subtractive primary dyes are generated during processing. Prior to this process, each colour record stores information in the form of a 'latent' silver image that catalyses the formation of the visible dyes. Reddish light at 600-700 nm gives a cyan dye, greenish light at 500-600 nm gives a magenta dye and bluish light at 400-500 $\mathrm{nm}$ gives a yellow dye [4].

The colour formation process is quite complex, as the dyes are formed "in situ" during the processing itself. In particular, the colour is given by the reaction between an oxidized developer, which is generally a derivative of $p$-phenylenediamine, and a dye-forming material or "coupler" to obtain magenta, cyan, or yellow colouring compounds.

A wide variety of couplers can be used, although manufacturers provide very little information on their precise nature. Generally, in the chromogenic films the magenta and yellow dyes belong to the family of azomethines, while the cyan dyes belong to that of indoanilines. In particular, the most important classes of yellow-forming couplers are $\alpha$-benzoylacetanilides and $\alpha$-pivaloylacetanilides. For magenta-forming couplers the main classes are 5-pyrazolone, indazolone, pyrazolobenzimidazole, and pyrazolotriazole. Finally, all the couplers that form cyan are of the families of phenol or naphthol, subsequently joined by 2-aryl-5-(arylamido)phenols due to their higher stability and superior colour properties [2,5] and even more recently by pyrrolotriazoles and pyrazolotriazoles [4].

Although extensive studies have reported on the performances and the degradation processes affecting these dyes [2,5], as mentioned above, the analytical detection of the molecules responsible for colour in a film is still an open challenge. In this work three dyes, representative of the most common classes in chromogenic films, were synthesised and characterised. In particular, analytical methods based on Raman spectroscopy were investigated as a means of identifying such dyes. Raman spectroscopy is a common tool in the field of diagnostics of cultural heritage materials, thanks to its specificity and potential non-invasiveness. However, when applied for the detection of organic compounds, it suffers from the problem of fluorescence emission associated with these molecules. Recently, the development of the innovative SSETM (Sequentially Shifted Excitation) strategy has made it possible to solve, or at least limit, this problem [6]. On the other hand, surfaceenhanced Raman spectroscopy (SERS) allows one to take advantage of the increased Raman signal of an analyte, when it is absorbed, or in proximity of, a rough metal surface. Thanks to this close contact, Raman scattering excited by visible light can experience an enhancement of up to a factor of $10^{8}$ [7] allowing the detection of analytes even at very low concentration. Furthermore, metal nanoparticles absorb most of the fluorescence photons emitted by molecules, acting as a quencher of the fluorescence emission often associated with organic substances. Therefore, SERS spectroscopy has been extensively exploited in the field of cultural heritage diagnostics for the detection of natural and synthetic dyes in fabrics and lakes. [8-12] The most typical SERS substrates are colloidal suspensions of metal nanoparticles, as they can be prepared quickly and easily used. This traditional approach involves the analysis in solution of the target analyte. Typically, the materials chosen for this purpose are spherical silver nanoparticles, prepared as a colloidal dispersion by the Lee-Meisel method [13]. More recently, it has been demonstrated that nanomaterials with anisotropic structure have shown greater efficiency in producing significant SERS enhancements [14-17]. For this reason, a silver nanostar colloid was used as SERS substrate, in the present work.

In the present work, three dyes have been synthesised as references, one for each of the three colours magenta, yellow, and cyan. In particular, a benzoylacetanilide, a pyrazolone, and a substituted phenol were selected as couplers for the yellow, magenta, and cyan dye, respectively. $N, N$-diethylbenzene-1,4-diamine was used to reproduce the role of the developer [18]. The synthesised compounds have, to the best of our knowledge, been successfully studied for the first time using Raman and SERS techniques and the results have been exploited to characterise dyes extracted from a real motion picture film. Preliminary results were promising, particularly those obtained by SERS spectroscopy, revealing a new potential application of this technique in the field of conservation science. 


\section{Materials and Methods}

\subsection{Synthesis of Dyes}

The dyes used as references for yellow (1), magenta (2), and cyan (3) colour (Figure 1) were prepared according to the procedures reported in the literature [18-22], as described in the Supporting Information (see the Word file "Synthesis and NMR characterisation of the reference dyes"). The molecular structures of the synthesized compounds were checked by recording their ${ }^{1} \mathrm{H}$ and ${ }^{13} \mathrm{C}$ NMR spectra (Word file "Synthesis and NMR characterisation of the reference dyes" Supporting Information).<smiles>CCN(CC)c1ccc(/N=C(/C(=O)Nc2ccccc2)C(=O)c2ccccc2)cc1</smiles>

1

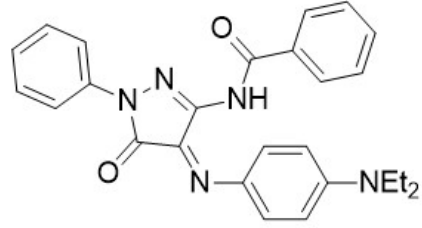

2

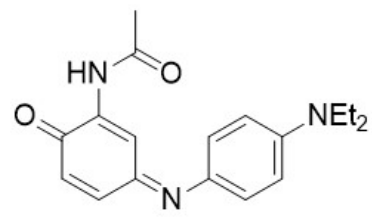

3

Figure 1. Structure of the dyes used as reference for yellow (1), magenta (2) and cyan (3) colour.

\subsection{Synthesis of Silver Nanostars}

Silver nanostars were synthesised according to the procedure reported by GarciaLeis [16]. Briefly, $500 \mu \mathrm{L}$ of a $0.05 \mathrm{M} \mathrm{NaOH}$ solution was stirred at $670 \mathrm{rpm}$ for one minute with $500 \mu \mathrm{L}$ of $50 \% w / v$ hydroxylamine solution. A total $9 \mathrm{~mL}$ of a $10^{-3} \mathrm{M} \mathrm{AgNO}_{3}$ solution was then added and the mixture was stirred for $5 \mathrm{~min}$. Finally, $100 \mu \mathrm{L}$ of a $1 \%$ trisodium citrate solution $(0.045 \mathrm{M})$ was dropped into the flask and stirring continued for $15 \mathrm{~min}$. The resulting dark green colloid was stored overnight in the dark before use to allow the nanoparticles to develop their final branched shape. The successful synthesis of star-shaped nanoparticles was verified by UV-Visible spectroscopy. An absorption band at $380 \mathrm{~nm}$, caused by the central core, was followed by a progressive increase in the NIR region (from about $700 \mathrm{~nm}$ ) produced by the several tips (Figure S1 Supporting Information) [17].

\subsection{Sample Preparation}

As a real case study, a $35 \mathrm{~mm}$ Fujicolour Positive Film Eterna-CP 3513DI was examined, containing the trailer for the animated film Happy Feet (Warner Bros, Burbank, CA, USA, 2006). In short, this film consists of a polyethylene terephthalate (PET) support, on which there are the three layers of light-sensitive emulsion, containing from top to bottom the magenta, cyan, and yellow dyes, isolated by suitable separatory layers and covered with a final protective coating [23].

For the analyses, the surface coating was removed, and the underlying part of the film was scratched with a scalpel in order to separate from the support all the layers of emulsion containing the colourants. The sample obtained was dispersed in a 3:1 of ethanol/water solution and the dyes were extracted under magnetic stirring for 2 days. The suspension was then centrifuged for $20 \mathrm{~min}$ at $6000 \mathrm{rpm}$ and the supernatant was collected, leaving the solid residue at the bottom of the centrifuge tube. The dyes were separated by thin layer chromatography (TLC). An ALUGRAM(R) Xtra SIL G/UV plate with a silica stationary phase was used, while the mobile phase was a mixture of diethyl ether and hexane (ratio 1:1). After separation, the spots corresponding to each dye (Figure S2 Supporting Information) were scratched away from the plate and the analytes were extracted from silica by sonication in ethanol. The extraction procedure was used because in a previous attempt of a TLC-SERS analysis, carried out by depositing few microlitres of pre-concentrated nanostars on the spots [24], the signals due to the colloid were the predominant ones. It should be noted that only one spot was obtained for magenta and yellow dyes respectively, while two cyan-coloured spots were observed. In principle, more than one dye for a given colour may be contained in the film and degradation products can also be observed in an aged film [2]. However, the latter hypothesis can probably be 
discarded as cyan dyes are the most stable and the degradation products formed by their residual couplers are generally colourless [2]. Of the two cyan spots, the extract obtained from the one better separated from those due to the other dyes was examined by SERS.

\subsection{SERS and Raman Analysis}

SERS analyses were performed on the reference dyes in ethanolic solution $(10 \mathrm{mM})$ or on the extracts obtained from the film. A total $200 \mu \mathrm{L}$ of silver nanostars were stirred on a magnetic plate, then $10 \mu \mathrm{L}$ of the dye solution was added. Although the activation by aggregation is not necessary for these nanoparticles due to the large enhancement provided by their special morphology, it was found that more intense SERS spectra are obtained if $5 \mu \mathrm{L}$ of sodium perchlorate $(1.8 \mathrm{M})$ is added to the nanoparticles before the analyte. A micro-drop of the final suspension was deposited on a glass slide and SERS analyses were performed using a JASCO RMP 100 portable Raman micro-probe. The micro-probe is equipped with an 1800 lines/mm grating, a notch filter, an Olympus 50 $\times$ microscope objective and a Peltiercooled CCD detector. A Nd:YAG laser delivered the exciting radiation at $532 \mathrm{~nm}$, with an output laser power of approximately $100 \mathrm{~mW}$. All SERS spectra were recorded between 2000 and $200 \mathrm{~cm}^{-1}$ as a sum of 30 scans with an exposure time of $2 \mathrm{~s}$.

Normal Raman analyses were performed directly on the powdered dye for the reference compounds. A Bruker BRAVO handheld spectrometer was used for the measurements. This instrument is based on the patented SSE ${ }^{\mathrm{TM}}$ technology, which involves the excitation of spectra by means of two diode lasers operating at different temperature and emitting respectively at 785 and $850 \mathrm{~nm}$. A suitable algorithm, therefore, allows to extract the final Raman spectral data. The spectra are collected in two sequential steps, from $300 \mathrm{~cm}^{-1}$ to $2000 \mathrm{~cm}^{-1}$ and from $2000 \mathrm{~cm}^{-1}$ to $3200 \mathrm{~cm}^{-1}$. The average spectral resolution is approximately $11 \mathrm{~cm}^{-1}$ and the applied laser power is always less than $100 \mathrm{~mW}$ for both lasers. The acquisition time varies from $500 \mathrm{~ms}$ to $2 \mathrm{~s}$ and the number of accumulations from 5 to 300 (these parameters are automatically set by the instrument).

\section{Results and Discussion}

The dyes synthesised as references were initially studied. The Raman spectra show peculiar pattern for each dye, characterised by intense and well-defined signals (Figure 2). The SSE ${ }^{\mathrm{TM}}$ technology, in fact, allows to strongly reduce the fluorescence background associated with these organic molecules.

Satisfying results were obtained also by SERS spectroscopy upon visible excitation at $532 \mathrm{~nm}$ (Figure 3). The interaction between the dye molecules and the silver nanostars, in fact, leads to the enhancement of the Raman scattering and to the quenching of the fluorescence emission. As expected, a correspondence was found between SERS and Raman spectra of the same dyes, despite small shifts of band positions and variations in the intensity ratios (Figure S3 Supporting Information). Tentative assignment of Raman and SERS bands are reported in Table 1 . The main SERS signals are attributable to the vibrational modes of the $\mathrm{C}=\mathrm{O}, \mathrm{C}=\mathrm{C}$, and $\mathrm{C}=\mathrm{N}$ bonds, as shown in the table. Furthermore, for the magenta dye 2 the bands assigned to the $\beta(\mathrm{C}-\mathrm{H})$ modes in the normal Raman spectra are absent in the SERS spectra, thus suggesting that the dye molecule adopts a flat-on geometry with respect to the silver nanoparticles and the main interaction between the molecule and the nanoparticles occurs through the conjugated system [25]. In fact, the substantial coplanarity of the phenyl, pyrazolone, and p-diethylaminophenyl rings was demonstrated in the literature by crystallographic studies for a molecule containing a methyl group instead of the benzoyl amide group [26]. It is interesting to note, however, that the azomethine yellow dyes belonging to the class of the compound synthesized in the present work are known to have generally less planar molecular structures [4]. In particular, for a molecule similar to 1, except for a methyl group on the p-diethylaminophenyl ring and a chlorine atom on the phenyl ring of the carbamoyl moiety, crystallographic studies have shown that the p-aminophenyl fragment is coplanar with the azomethine-carbamoyl fragment, but is perpendicular to the acyl one [27]. Consistently, in the SERS spectrum 
of the synthesized yellow dye the strongest bands are again due to vibrational modes of $\mathrm{C}=\mathrm{C}$ bonds, but a $\beta(\mathrm{C}-\mathrm{H})$ band with medium intensity is still observed at $1172 \mathrm{~cm}^{-1}$ (Table 1). This fact, coupled with the unshifted position of the carbonyl band, suggests that the interaction still takes place through the $\pi$ system, but with a tilted orientation of the molecule relative to the silver nanoparticles. A similar situation probably takes place for the indoaniline cyan dye 3 , for whose molecule a dihedral angle of $47^{\circ}$ has been calculated between the quinone and the aniline rings [28]. In the corresponding SERS spectrum, the band due the $\mathrm{C}=\mathrm{O}$ group of indoaniline at $1630 \mathrm{~cm}^{-1}$ is still intense, but does not show any shift possibly associated with an interaction with the silver nanoparticles through the lone pairs of the oxygen atom. Furthermore, two particularly strong bands are located at 791 and $433 \mathrm{~cm}^{-1}$ and have been tentatively assigned to the 10a and 16a modes of the diethylaniline ring (Table 1), both corresponding to out-of-plane vibrational modes in the Wilson notation [29]. On the other hand, a band possibly associated to the in-plane 9a mode also appears with medium intensity at $1191 \mathrm{~cm}^{-1}$. In summary, an interaction through the double-bond system, with a tilted orientation of the molecule, seems the most probable also for the cyan dye, despite the fact that the $\pi$-conjugation is limited due to the lack of perfect planarity.

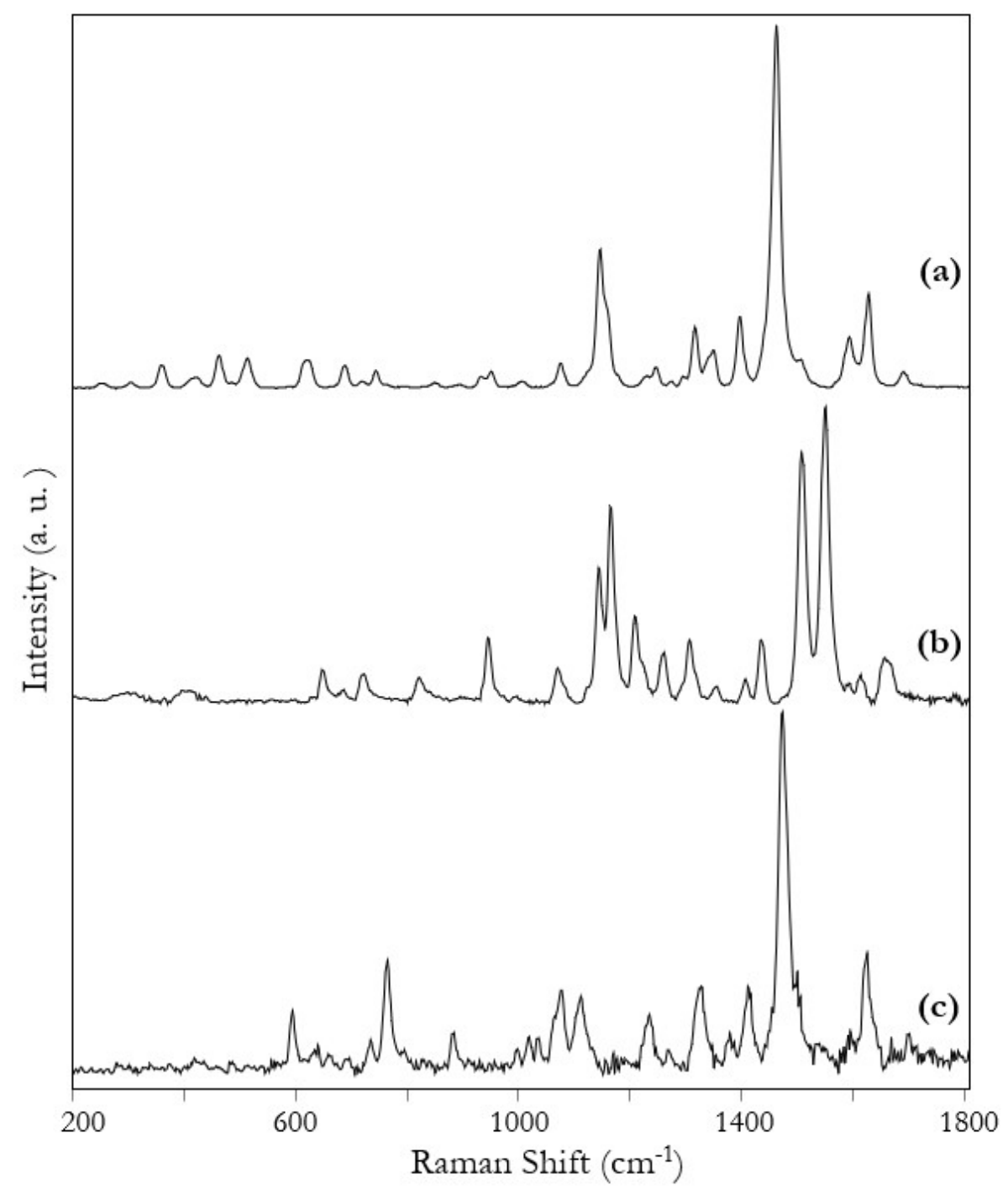

Figure 2. SSE ${ }^{\mathrm{TM}}$ Raman spectra of pure powdered (a) cyan, (b) yellow and (c) magenta dyes. 


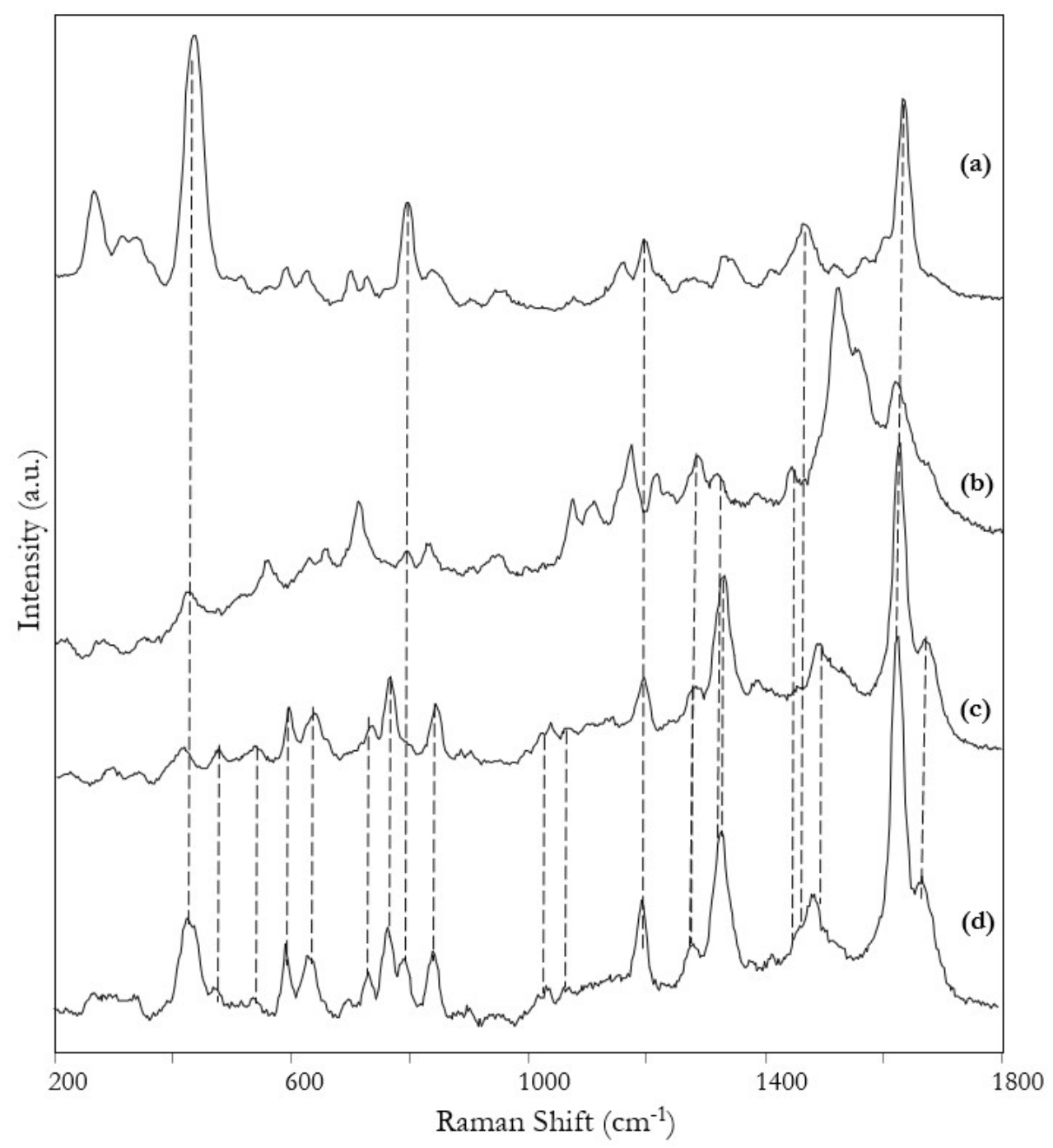

Figure 3. SERS spectra of (a) cyan, (b) yellow, (c) magenta, and (d) a mixture 1:1:1 of the three dyes. Spectra were obtained from $10 \mathrm{mM}$ ethanolic solutions.

Table 1. Tentative assignment of Raman and SERS bands for the reference dyes magenta, yellow, and cyan.

\begin{tabular}{ccc}
\hline Raman & SERS & Tentative Assignment \\
\hline Magenta [30-33] & & $v(\mathrm{C}=\mathrm{O})$ amide \\
$1699 \mathrm{w}$ & - & $v(\mathrm{C}=\mathrm{O})$ pyrazolone $+v(\mathrm{C}=\mathrm{N})$ \\
$1667 \mathrm{w}$ & $1667 \mathrm{~m}$ & $v(\mathrm{C}=\mathrm{C})+v(\mathrm{C}=\mathrm{N})$ \\
$1624 \mathrm{~m}$ & $1624 \mathrm{~s}$ & $\nu(\mathrm{C}=\mathrm{C})$ \\
$1473 \mathrm{vs}$ & $1484 \mathrm{mw}$ & $\beta(\mathrm{C}-\mathrm{H})$ \\
$1411 \mathrm{~m}$ & - & $\beta(\mathrm{C}-\mathrm{H})$ \\
$1325 \mathrm{~m}$ & $1325 \mathrm{~ms}$ & $\beta(\mathrm{C}-\mathrm{H})$ \\
$1230 \mathrm{~m}$ & - & $\beta(\mathrm{C}-\mathrm{H})$ \\
- & $1194 \mathrm{mw}$ & $\gamma(\mathrm{C}-\mathrm{H})$ \\
$1110 \mathrm{~m}$ & - & $\gamma(\mathrm{C}-\mathrm{H})$ \\
$1076 \mathrm{~m}$ & - & $\beta(\mathrm{C}-\mathrm{H})$ ring $+\gamma(\mathrm{CNN})$ \\
$883 \mathrm{mw}$ & - & pyrazolone ring stretching $+\beta(\mathrm{C}-\mathrm{H})$ \\
- & $840 \mathrm{mw}$ & reformation \\
$765 \mathrm{~m}$ & $765 \mathrm{mw}$ & aromatic and heterocyclic ring deformation \\
$638 \mathrm{w}$ & $638 \mathrm{mw}$ & aromatic and heterocyclic ring deformation \\
$595 \mathrm{mw}$ & $595 \mathrm{mw}$ &
\end{tabular}


Table 1. Cont.

\begin{tabular}{|c|c|c|}
\hline Raman & SERS & Tentative Assignment \\
\hline $1670,1660 \mathrm{mw}$ & $1670,1660 \mathrm{sh}$ & $v(\mathrm{C}=\mathrm{O})$ amide, $v(\mathrm{C}=\mathrm{N})$ \\
\hline $1619 \mathrm{w}$ & $1620 \mathrm{~m}$ & $v(C=C)$ \\
\hline 1555 vs & $1550 \mathrm{~ms}$, sh & $v(\mathrm{C}=\mathrm{C})+\beta(\mathrm{N}-\mathrm{H})$ \\
\hline 1513 vs & 1515 vs & $v(C=C)$ \\
\hline $1440 \mathrm{mw}$ & $1440 \mathrm{w}$ & $v(\mathrm{C}=\mathrm{C})$ \\
\hline $1311 \mathrm{mw}$ & $1316 \mathrm{w}$ & $v(C=C)$ \\
\hline- & $1284 \mathrm{mw}$ & - \\
\hline $1266 \mathrm{mw}$ & $1269 \mathrm{sh}$ & - \\
\hline $1214 \mathrm{~m}$ & $1214 \mathrm{mw}$ & - \\
\hline $1170 \mathrm{~s}$ & $1172 \mathrm{~m}$ & $\beta(\mathrm{C}-\mathrm{H})$ \\
\hline $1150 \mathrm{~s}$ & $1151 \mathrm{sh}$ & $\beta(\mathrm{C}-\mathrm{H})$ \\
\hline- & $1110 \mathrm{w}$ & - \\
\hline $1075 \mathrm{w}$ & $1073 \mathrm{w}$ & $\beta(\mathrm{C}-\mathrm{H})$ \\
\hline $950 \mathrm{mw}$ & $950 \mathrm{mw}$ & $v(C=C)$ \\
\hline $828 w$ & $828 \mathrm{w}$ & $\beta(\mathrm{C}=\mathrm{O})$ anilide? \\
\hline- & $712 \mathrm{~m}$ & $\gamma(\mathrm{N}-\mathrm{H}) ?$ \\
\hline \multicolumn{3}{|l|}{ Cyan $[30,35,36]$} \\
\hline $1683 \mathrm{w}$ & - & $v(\mathrm{C}=\mathrm{O})$ amide \\
\hline $1628 \mathrm{~m}$ & $1630 \mathrm{~s}$ & $v(\mathrm{C}=\mathrm{O})$ indoaniline \\
\hline $1594 \mathrm{mw}$ & $1601 \mathrm{sh}$ & $8 \mathrm{a}^{\text {a }}$ diethylaniline \\
\hline- & $1567 \mathrm{sh}$ & $v(\mathrm{C}=\mathrm{C})$ \\
\hline $1511 \mathrm{sh}$ & $1514 \mathrm{w}$ & $v(\mathrm{C}=\mathrm{N})$ \\
\hline 1464 vs & $1464 \mathrm{~m}$ & $\vee(C=C) ?$ \\
\hline $1401 \mathrm{~m}$ & $1401 \mathrm{vw}$ & $\delta(\mathrm{C}-\mathrm{H})$ ethyl? \\
\hline $1352 \mathrm{mw}$ & - & $\delta(\mathrm{C}-\mathrm{H})$ ethyl? \\
\hline- & $1340 \mathrm{mw}$ & $v(\mathrm{C}-\mathrm{N})$ \\
\hline $1321 \mathrm{~m}$ & $1327 \mathrm{mw}$ & - \\
\hline- & $1191 \mathrm{~m}$ & $9 \mathrm{a}^{\mathrm{a}}$ diethylaniline? \\
\hline $1150 \mathrm{~s}$ & $1153 \mathrm{~m}$ & $v(\mathrm{C}-\mathrm{N})$ \\
\hline- & $791 \mathrm{~ms}$ & $10^{\text {a }}$ diethylaniline? \\
\hline $428 \mathrm{w}$ & $433 \mathrm{vs}$ & $16 \mathrm{a}^{\mathrm{a}}$ diethylaniline? \\
\hline
\end{tabular}

${ }^{a}$ Numbers refer to the Wilson notation.

It is worth noting that, on the other hand, normal Raman spectroscopy with visible excitation $(532 \mathrm{~nm})$ did not lead to significant results as almost no signals due to the any of the three dyes could be recognised, and only those due to the glass slide were observed (Figure S4 Supporting Information).

In addition, a 1:1:1 mixture of the three dyes was analysed. The acquired SERS spectrum (Figure 3d), although the relative concentration is the same for each dye, is dominated by the signals due to magenta, and only minor features are attributable to yellow and cyan dyes. This is probably due to the fact that the red hue of this dye fulfils the resonance conditions with the green laser beam.

SERS analysis was then applied to the dyes extracted from the film, before and after TLC separation. It should be noted that the low amount of dye extracted for each colour prevented the acquisition of SSE ${ }^{\mathrm{TM}}$ spectra, as the instrumentation used did not allow a microanalysis. On the other hand, SSE ${ }^{\mathrm{TM}}$ measurements were also performed directly on differently coloured frames of the film (results not shown), but of course the obtained spectra were complicated by the bands due to the polymer and by the superposition of bands due to the different dyes. As shown in Figure 4, good-quality SERS spectra were obtained from each dye and were compared with those of the reference compounds. 


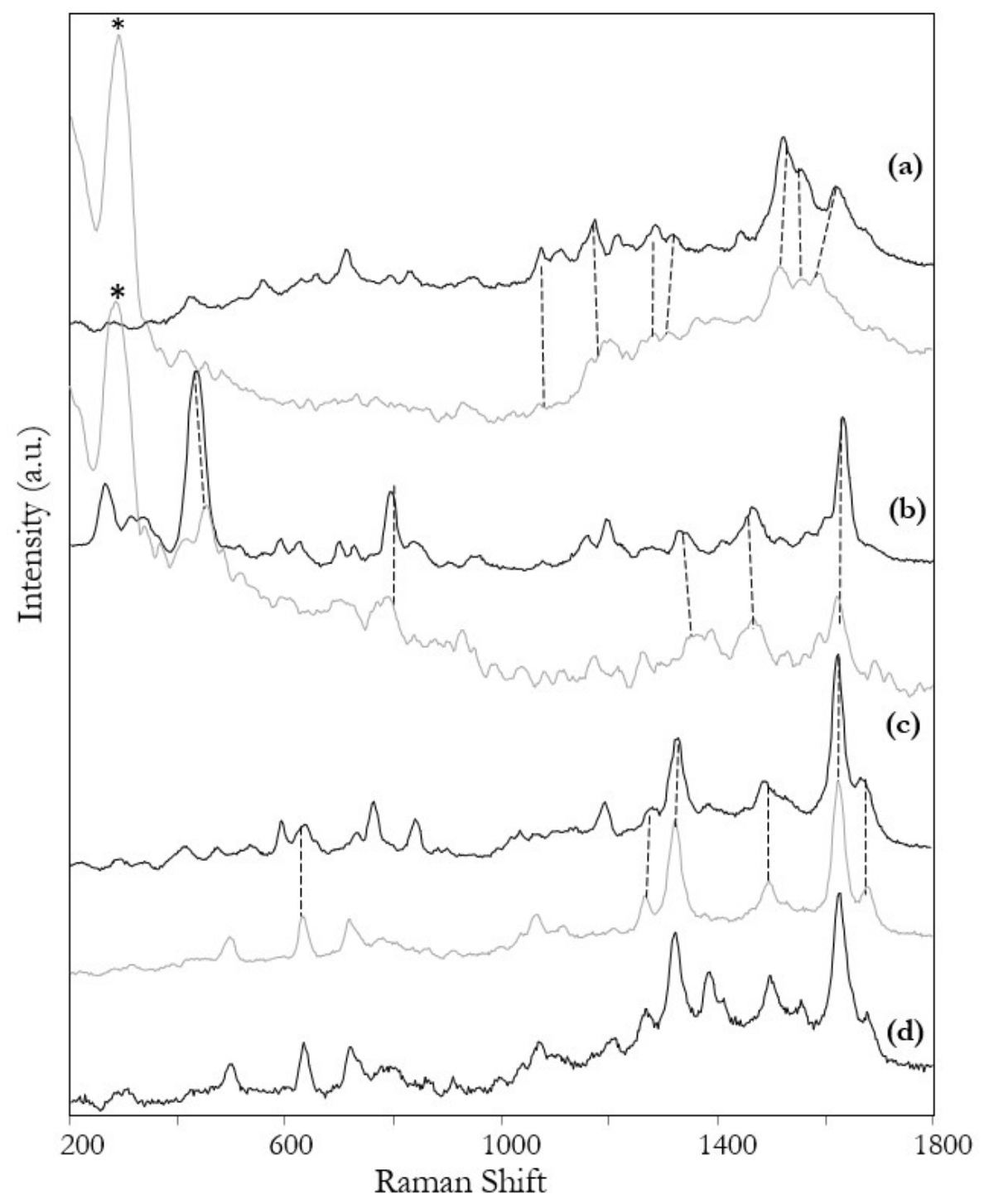

Figure 4. SERS spectra of (a) yellow, (b) cyan, (c) magenta dyes extracted after TLC separation (grey line) in comparison with those of the synthesised reference colourants (black line) and (d) SERS spectrum of the overall extract from the motion picture film. ${ }^{*}=$ signal due to silver colloid.

The similarity between the spectral patterns suggests that the dyes in the film belong to the same chemical class as the reference ones, at least as regards the core of the molecules. On the other hand, it should be remembered that the true dyes in the chromogenic films are much larger molecules than the reference ones. This is due to the presence of substituents that have the role of "ballast", i.e., useful to retain the couplers in their coating layer $[4,5]$ and to avoid their migration in other layers. Such substituents can be for example hydrophobic groups or polymeric chains [2].

Thanks to its selectivity towards the conjugated core of the molecules, SERS spectroscopy can be more effective in the classification of these dyes compared to other molecular analysis techniques, such as infrared spectroscopy. This information is relevant because, in any case, most of the degradation processes that must be considered in the conservation and restoration of the films are mainly related to the molecular classes to which the couplers belong.

\section{Conclusions}

The present work aimed at a preliminary investigation on the possibility of applying SERS spectroscopy to the classification of dyes in chromogenic films, to obtain information that may be used for the purposes of conservation and digital restoration. 
Reference dyes deriving from three of the most common classes of couplers used in such films were synthesized and characterized for the first time by normal Raman and SERS spectroscopy. Interpretation of SERS spectra suggested that the main interaction between the molecules and the silver nanoparticles occurred through the conjugated $\pi$ system.

The comparison between the SERS spectra of the reference compounds and those of dyes extracted from a motion picture film and separated by TLC indicated that such dyes most likely belong to the same chemical classes as the reference ones. SERS spectroscopy can therefore be proposed as a promising method to individuate these classes, each having peculiar features from the point of view of degradation mechanisms.

Naturally, this preliminary study should be extended to a wider number of reference compounds, representative of other types of couplers, and of dyes extracted from films of different brands and datable to different periods.

Supplementary Materials: The following supporting information can be downloaded at: https:/ / www.mdpi.com/article/10.3390/chemosensors10030101/s1, Synthesis and NMR characterisation of the reference dyes; Figure S1: UV-visible spectrum of the silver nanostar colloid; Figure S2: TLC separation of the extract from the emulsion obtained from the Fujicolour positive film; Figure S3: Comparison between SERS (black line) and SSE ${ }^{\mathrm{TM}}$ Raman (grey line) spectra of (a) cyan, (b) yellow and (c) magenta reference dyes. The SERS spectra were obtained from $10 \mathrm{mM}$ ethanolic solutions, the Raman ones from the pure powder dyes. Figure S4: Normal Raman spectra $\left(\lambda_{\text {exc }}=532 \mathrm{~nm}\right)$ obtained from ethanolic solution (10 mM) of the (a) cyan, (b) yellow and (c) magenta reference dyes. * = signals due to glass.

Author Contributions: Conceptualization, S.B. and M.L.; methodology, S.B., M.L. and F.F.; formal analysis, S.B., M.L. and F.F.; investigation, M.L., S.Z., L.C. and F.F.; resources, S.B. and F.F.; data curation, S.B., M.L., F.F., S.Z. and L.C.; writing-original draft preparation, M.L. and F.F.; writingreview and editing, S.B., M.L. and F.F.; visualization, M.L., S.Z. and F.F.; supervision, S.B. and F.F. All authors have read and agreed to the published version of the manuscript.

Funding: This research received no external funding.

Institutional Review Board Statement: Not applicable.

Informed Consent Statement: Not applicable.

Data Availability Statement: Not applicable.

Acknowledgments: Alessandro Rizzi, Alice Plutino and Beatrice Sarti (Department of Computer Science, University of Milan, Italy) are acknowledged for inspiring this research and for supplying the Fuji motion picture film.

Conflicts of Interest: The authors declare no conflict of interest.

\section{References}

1. Timeline of Historical Film Colors. Available online: https:/ / filmcolors.org (accessed on 18 January 2022).

2. Di Pietro, G. Examples of using advanced analytical techniques to investigate the degradation of photographic materials. In Physical Techniques in the Study of Art, Archaeology and Cultural Heritage; Creagh, D., Bradley, D., Eds.; Elsevier: Amsterdam, The Netherlands, 2007; pp. 155-197.

3. Lima da Silva, J.S. Ângelo de Sousa's Photographic and Film Collection: Strategies for the Preservation of Colour Slide-Based Artworks. Ph.D. Thesis, Universidade Nova de Lisboa, Lisbon, Portugal, 2019.

4. Friedrich, L.E.; Capecki, J.A. Color-Forming Photographic Materials. In Handbook of Imaging Materials, 2nd ed.; Diamond, A.S., Weiss, D.S., Eds.; Marcel Dekker, Inc.: New York, NY, USA, 2002; pp. 35-62.

5. Theys, R.D.; Sosnovsky, G. Chemistry and Processes of Color Photography. Chem. Rev. 1997, 97, 83-132. [CrossRef] [PubMed]

6. Conti, C.; Botteon, A.; Bertasa, M.; Colombo, C.; Realini, M.; Sali, D. Portable Sequentially Shifted Excitation Raman spectroscopy as an innovative tool for in situ chemical interrogation of painted surfaces. Analyst 2016, 141, 4599-4607. [CrossRef] [PubMed]

7. Jeanmaire, D.L.; Van Duyne, R.P. Surface Raman Spectroelectrochemistry Part1. Heterocyclic, aromatic and aliphatic amines adsorbed on the anodized silver electrode. J. Electroanal. Chem. 1977, 84, 1-20. [CrossRef]

8. Pozzi, F.; Leona, M. Surface-enhanced Raman spectroscopy in art and archaeology. J. Raman Spectrosc. 2016, 47, 67-77. [CrossRef]

9. Chen, K.; Leona, M.; Vo-Dinh, T. Surface-enhanced Raman scattering for identification of organic pigments and dyes in works of art and cultural heritage material. Sens. Rev. 2007, 27, 109-120. [CrossRef]

10. Casadio, F.; Leona, M.; Lombardi, J.R.; Van Duyne, R. Identification of organic colorants in fibers, paints, and glazes by surface enhanced Raman spectroscopy. Acc. Chem. Res. 2010, 43, 782-791. [CrossRef] [PubMed] 
11. Leona, M.; Decuzzi, P.; Kubic, T.A.; Gates, G.; Lombardi, J.R. Nondestructive identification of natural and synthetic organic colorants in works of art by surface enhanced Raman scattering. Anal. Chem. 2011, 83, 3990-3993. [CrossRef]

12. Leona, M.; Stenger, J.; Ferloni, E. Application of surface-enhanced Raman scattering techniques to the ultrasensitive identification of natural dyes in works of art. J. Raman Spectrosc. 2006, 37, 981-992. [CrossRef]

13. Lee, P.C.; Meisel, D. Adsorption and surface-enhanced Raman of dyes on silver and gold sols. J. Phys. Chem. 1982, 86, 3391-3395. [CrossRef]

14. Guerrero-Martínez, A.; Barbosa, S.; Pastoriza-Santos, I.; Liz-Marzán, L.M. Nanostars shine bright for you. Curr. Opin. Colloid Interface Sci. 2011, 16, 118-127. [CrossRef]

15. Wang, Y.; Camargo, P.H.C.; Skrabalak, S.E.; Gu, H.; Xia, Y. A Facile, Water-Based Synthesis of Highly Branched Nanostructures of Silver. Langmuir 2008, 24, 12042-12046. [CrossRef] [PubMed]

16. Garcia-Leis, A.; Garcia-Ramos, J.V.; Sanchez-Cortes, S. Silver Nanostars with High SERS Performance. J. Phys. Chem. C 2013, 117, 7791-7795. [CrossRef]

17. Zalaffi, M.S.; Litti, L.; Canton, P.; Meneghetti, M.; Moretto, L.M.; Ugo, P. Preparation and characterization of Ag-nanostars@Aunanowires hierarchical nanostructures for highly sensitive surface enhanced Raman spectroscopy. Nano Express 2020, 1, 020006 [CrossRef]

18. Reijers, E.B. How to Preserve Photographic Artworks for the Future: Chemical and Physical Interactions and Implications for Conservation Strategies. Ph.D. Thesis, Utrecht University, Utrecht, The Netherlands, 2017.

19. Sano, K. Alkaline hydrolysis of yellow azomethine dyes. J. Org. Chem. 1969, 34, 2076-2080. [CrossRef]

20. Vittum, P.W.; Duennebier, F.C. The Reaction between Pyrazolones and their Azomethine Dyes. J. Am. Chem. Soc. 1950, 72, 1536-1538. [CrossRef]

21. Cusan, C.; Spalluto, G.; Prato, M.; Adams, M.; Bodensieck, A.; Bauer, R.; Tubaro, A.; Bernardi, P.; Da Ros, T. Synthesis and biological evaluation of a new class of acyl derivatives of 3-amino-1-phenyl-4,5-dihydro-1H-pyrazol-5-one as potential dual cyclooxygenase (COX-1 and COX-2) and human lipoxygenase (5-LOX) inhibitors. Il Farmaco 2005, 60, 327-332. [CrossRef] [PubMed]

22. Vittum, P.W.; Brown, G.H. Indoaniline Dyes. I. Some Phenol Blue Derivatives with Substituents in the Phenol Ring. J. Am. Chem. Soc. 1946, 68, 2235-2239. [CrossRef]

23. Fujifilm: Value from Innovation. Available online: https:/ /www.fujifilm.com (accessed on 18 January 2022).

24. Zalaffi, M.S.; Agostinelli, I.; Karimian, N.; Ugo, P. Ag-nanostars for the sensitive SERS detection of dyes in artistic cross-sectionsMadonna della Misericordia of the National Gallery of Parma: A case study. Heritage 2020, 3, 74. [CrossRef]

25. Gao, X.; Davies, J.P.; Weaver, M.J. A test of surface selection rules for surface-enhanced Raman scattering: The orientation of adsorbed benzene and monosubstituted benzenes on gold. J. Phys. Chem. 1990, 94, 6858-6864. [CrossRef]

26. Smith, D.L.; Barrett, E.K. The crystal structures of two pyrazolinone azomethine dyes. Acta Cryst. 1971, B27, 2043-2057. [CrossRef]

27. Ichijima, S.; Fukunaga, H.; Kobayashi, H.; Ishihara, M.; Kogay, N. Theoretical study on the structures and absorption properties of yellow azomethine dyes. Bull. Chem. Soc. Jpn. 2003, 76, 733-742. [CrossRef]

28. Adachi, M.; Murata, Y.; Nakamura, S. Theoretical and experimental studies of indoaniline dyes. A novel relationship between absorption spectra and molecular structure. J. Am. Chem. Soc. 1993, 115, 4331-4338. [CrossRef]

29. Gardner, A.M.; Wright, T.G. Consistent assignment of the vibrations of monosubstituted benzenes. J. Chem. Phys. 2011, 135, 114305. [CrossRef] [PubMed]

30. Socrates, G. Infrared and Raman Characteristic Group Frequencies, 3rd ed.; John Wiley \& Sons: Chichester, UK, 2001.

31. Alam, M.S.; Lee, D.-U. Spectral (FT-IR, FT-Raman, UV, and fluorescence), DFT, and solid state interaction analyses of (E)-4-(3,4dimethoxybenzylideneamino)-1,5-dimethyl-2-phenyl-1H-pyrazol-3(2H)-one. J. Mol. Struct. 2017, 1128, 174-185. [CrossRef]

32. Alam, M.S.; Lee, D.-U. Molecular structure, spectral (FT-IR, FT-Raman, Uv-Vis, and fluorescent) properties and quantum chemical analyses of azomethine derivative of 4-aminoantipyrine. J. Mol. Struct. 2021, 1227, 129512. [CrossRef]

33. Durig, J.R.; Mamula Bergana, M.; Zunic, W. Fourier transform Raman spectrum of polycrystalline pyrazole, vibrational assignment and ab initio calculations. J. Raman Spectrosc. 1992, 23, 157-363. [CrossRef]

34. Arjunan, V.; Kalaivani, M.; Senthilkumari, S.; Mohan, S. Vibrational, NMR and quantum chemical investigations of acetoacetanilde, 2-chloroacetoacetanilide and 2-methylacetoacetanilide. Spectrochim. Acta A 2013, 115, 154-174. [CrossRef]

35. Yamaguchi, T.; Kimura, Y.; Hirota, N. Solvent and solvent density effects on the spectral shifts and the bandwidths of the absorption and the resonance Raman spectra of Phenol Blue. J. Phys. Chem. A 1997, 101, 9050-9060. [CrossRef]

36. Brouwer, A.M.; Wilbrandt, R. Vibrational spectra of $N, N$-dimethylaniline and its radical cation. An interpretation based on quantum chemical calculations. J. Phys. Chem. 1996, 100, 9678-9688. [CrossRef] 